\title{
From ARB to ARNI in Cardiovascular Control
}

\author{
Estrellita Uijl $^{1,2}$ • Lodi C. W. Roksnoer ${ }^{1,2}$ - Ewout J. Hoorn ${ }^{2}$ • A. H. Jan Danser ${ }^{1}$
}

Published online: 11 November 2016

(C) The Author(s) 2016. This article is published with open access at Springerlink.com

\begin{abstract}
Coexistence of hypertension, diabetes mellitus and chronic kidney disease synergistically aggravates the risk of cardiovascular and renal morbidity and mortality. These highrisk, multi-morbid patient populations benefit less from currently available anti-hypertensive treatment. Simultaneous angiotensin II type 1 receptor blockade and neprilysin inhibition ('ARNI') with valsartan/sacubitril (LCZ696) might potentiate the beneficial effects of renin-angiotensin-aldosterone inhibition by reinforcing its endogenous counterbalance, the natriuretic peptide system. This review discusses effects obtained with this approach in animals and humans. In animal models of hypertension, either alone or in combination with myocardial infarction or diabetes, ARNI consistently reduced heart weight and cardiac fibrosis in a blood pressure-independent manner. Additionally, LCZ696 treatment reduced proteinuria, focal segmental glomerulosclerosis and retinopathy, thus simultaneously demonstrating favourable effects on microvascular complications. These results were confirmed in patient populations. Besides blood pressure reductions in hypertensive patients and greatly improved (cardiovascular) mortality in heart failure patients, ventricular wall stress and albuminuria were reduced particularly in diabetic patients. The exact underlying mechanism remains unknown, but may involve improved renal haemodynamics and reduced glomerulosclerosis, e.g. related to a
\end{abstract}

This article is part of the Topical Collection on Hypertension and the Kidney

A. H. Jan Danser

a.danser@erasmusmc.nl

1 Division of Pharmacology and Vascular Medicine Department of Internal Medicine, Erasmus MC, Rotterdam, The Netherlands

2 Division of Nephrology and Transplantation, Department of Internal Medicine, Erasmus MC, Rotterdam, The Netherlands rise in natriuretic peptide levels. However, the assays of these peptides are hampered by methodological artefacts. Moreover, since sacubitrilat is largely renally cleared, drug accumulation may occur in patients with impaired renal function and thus hypotension is a potential side effect in patients with chronic kidney disease. Further caution is warranted since neprilysin also degrades endothelin-1 and amyloid beta in animal models. Accumulation of the latter may increase the risk of Alzheimer's disease.

Keywords Diabetes · Neprilysin · Angiotensin · Natriuretic peptide $\cdot$ Chronic kidney disease

\section{Introduction}

Cardiovascular diseases cause nearly one third of all deaths worldwide [1], and $50 \%$ of these deaths are thought to be directly attributable to hypertension [2]. High blood pressure is an important risk factor in the development of myocardial infarction, hypertensive heart disease, heart failure and stroke. Additionally, hypertension may cause chronic kidney disease (CKD) and end-stage renal disease (ESRD). Patients with CKD are more likely to die from cardiovascular disease than to develop renal failure [3]. They are regarded as a high-risk population within the general hypertensive population. Another high-risk population comprises hypertensive patients with diabetes mellitus. Prevalence of hypertension among diabetic patients is reported in most studies to be greater than $60 \%$ and in many studies to be even higher than 75\% [4]. Coexistence of hypertension and diabetes synergistically aggravates the risk of developing macro- and microvascular complications [5]. These dismal outcomes emphasize the need for targeted therapy of these vulnerable multi-morbid patient populations. 
Treatment of high blood pressure greatly reduces the risk for all-cause mortality, coronary heart disease, heart failure and stroke. Development of renal failure, however, is not prevented by blood pressure lowering, and high-risk populations benefit less from this approach. Indeed, proportional risk reductions for major cardiovascular events are lower or absent in patients with concurrent diabetes or CKD [6]. However, treatment effects vary between different classes of antihypertensive drugs. Pharmacological inhibition of the reninangiotensin-aldosterone system (RAAS) reduces albuminuria and slows the progression of diabetic nephropathy [7]. Unfortunately, effects on cardiovascular events and all-cause mortality remain limited. Initially, it was believed that this was due to incomplete RAAS blockade and RAAS escape phenomena. Although the combination of angiotensin-converting enzyme inhibition (ACEi) and angiotensin receptor blockade (ARB) appears to prevent the development of ESRD more effectively according to one meta-analysis [7], dual therapy is not recommended [8]. The reason is that near complete RAAS suppression does not reduce cardiovascular or allcause mortality. Yet, it does increase the risk of adverse events, including hypotension, hyperkalaemia and acute kidney injury (AKI) [9]. This most likely relates to the fact that angiotensin II (Ang II) is essential to preserve renal function and glomerular filtration. The kidneys will do everything possible to keep the latter in the normal range, including massive upregulation of renin during RAAS blockade [10]. This mechanism is referred to as the 'nephrocentric' reaction to RAAS blockade in patients with heart failure [11]. In exceptional cases, particularly with drugs that accumulate in the kidney (such as renin inhibitors [12]), the nephrocentric reaction may even lead to extrarenal RAAS activation. The 8th Joint National Committee currently recommends ACEi or ARB monotherapy as first-line treatment for patients with CKD [8]. Clearly, rather than blocking the RAAS with two or more drugs, there is a need for interference with alternative systems. This review aims to critically evaluate the potential for cardiovascular control of a new anti-hypertensive treatment strategy, the combination of ARB and neprilysin inhibition: ARNI.

\section{Mechanism of Action}

Neprilysin, or neutral endopeptidase (NEP), is a transmembrane zinc-dependent metalloprotease with a molecular weight of approximately $85 \mathrm{kDa}$. Although expressed in many epithelial tissues, its levels are particularly high at the luminal side of the renal proximal tubule [13, 14]. NEP cleaves peptides and thereby inactivates peptide hormones such as glucagon, bradykinin, angiotensin, endothelin-1, substance $\mathrm{P}$, oxytocin, neurotensin, adrenomedullin and natriuretic peptides. Among these are both vasodilators (e.g. bradykinin, natriuretic peptides) and vasoconstrictors (e.g. angiotensin, endothelin-1). Therefore, the effect of NEP inhibition is ambiguous and depends on the relative dominance of the various substrates of NEP (Fig. 1). Consequently, NEP inhibition can even raise blood pressure, as shown in healthy men [15]. It was postulated that the combination of a NEP inhibitor with an ARB would hold the beneficial effects of increasing natriuretic peptides and simultaneously block the harmful effects of (the NEP inhibitor-induced increase in) Ang II. ARBs prevent the binding of Ang II to the Ang II type $1\left(\mathrm{AT}_{1}\right)$ receptor, thereby inhibiting Ang II-induced vasoconstriction, aldosterone release, stimulation of the sympathetic nervous system and vascular as well as cardiac remodeling [16]. Natriuretic peptides counter the RAAS not only by inducing natriuresis, diuresis and vasodilation but also by inhibiting renin secretion, via their second messenger cyclic guanosine 3'5'monophosphate (cGMP) [17]. Therefore, reinforcement of the natriuretic peptide system on top of RAAS blockade might have a synergistic effect on blood pressure and cardiac remodeling. LCZ696 (sacubitril/ valsartan, brand name Entresto) is the first-in-class ARNI. It is a single molecule in which the ARB valsartan and the NEP inhibitor prodrug sacubitril (AHU377) are combined, in a molar ratio of 1:1 [18]. Sacubitril is almost completely metabolized into its active form LBQ657 (also known as sacubitrilat) via ester hydrolysis, rapidly after ingestion [19]. In humans, sacubitril has a half life of $1.4 \mathrm{~h}$, LBQ657 has a half life of $11.5 \mathrm{~h}$ and valsartan has a half life of $9.9 \mathrm{~h}$. LBQ657 is excreted through urine, and the exposure to LBQ657 increases with decreasing renal function [20]. A decrease in renal function does not affect the pharmacokinetics of valsartan, since it is primarily excreted via the biliary route [21].

\section{Animal Studies}

Currently, the number of animal studies using ARNI is limited (Table 1). In 2008, Pu et al. compared the ARB valsartan with the ARNI combination valsartan + CGS25354 and a dual ACE inhibitor/NEP inhibitor in stroke-prone spontaneously hypertensive rats [22]. Compared with valsartan, both ARNI and the dual ACE/NEP inhibitor were more effective in lowering blood pressure and decreasing vascular remodeling and macrophage infiltration. The ACE/NEP inhibitor reduced cardiac hypertrophy more than ARNI, possibly because the ACE/NEP inhibitor also had a greater effect on blood pressure compared with ARNI [22]. Because the study did not include an ACE inhibition-only group, it is unclear if this was due to a difference in effectiveness between ARB and ACE inhibition 
Fig. 1 Opposing effects of neprilysin (NEP) inhibition. NEP degrades vasodilators (e.g. A-type (atrial) natriuretic peptide (ANP) produced by atrial myocytes, Btype (brain) natriuretic peptide (BNP) produced by ventricular myocytes and urodilatin produced by the distal convoluted tubule and the collecting duct) as well as vasoconstrictors (e.g. angiotensin II (Ang II) and endothelin-1 produced by endothelial cells) into inactive metabolites. The effect of NEP inhibition with LCZ696 may be unpredictable as it depends on the relative dominance of either vasodilators or vasoconstrictors

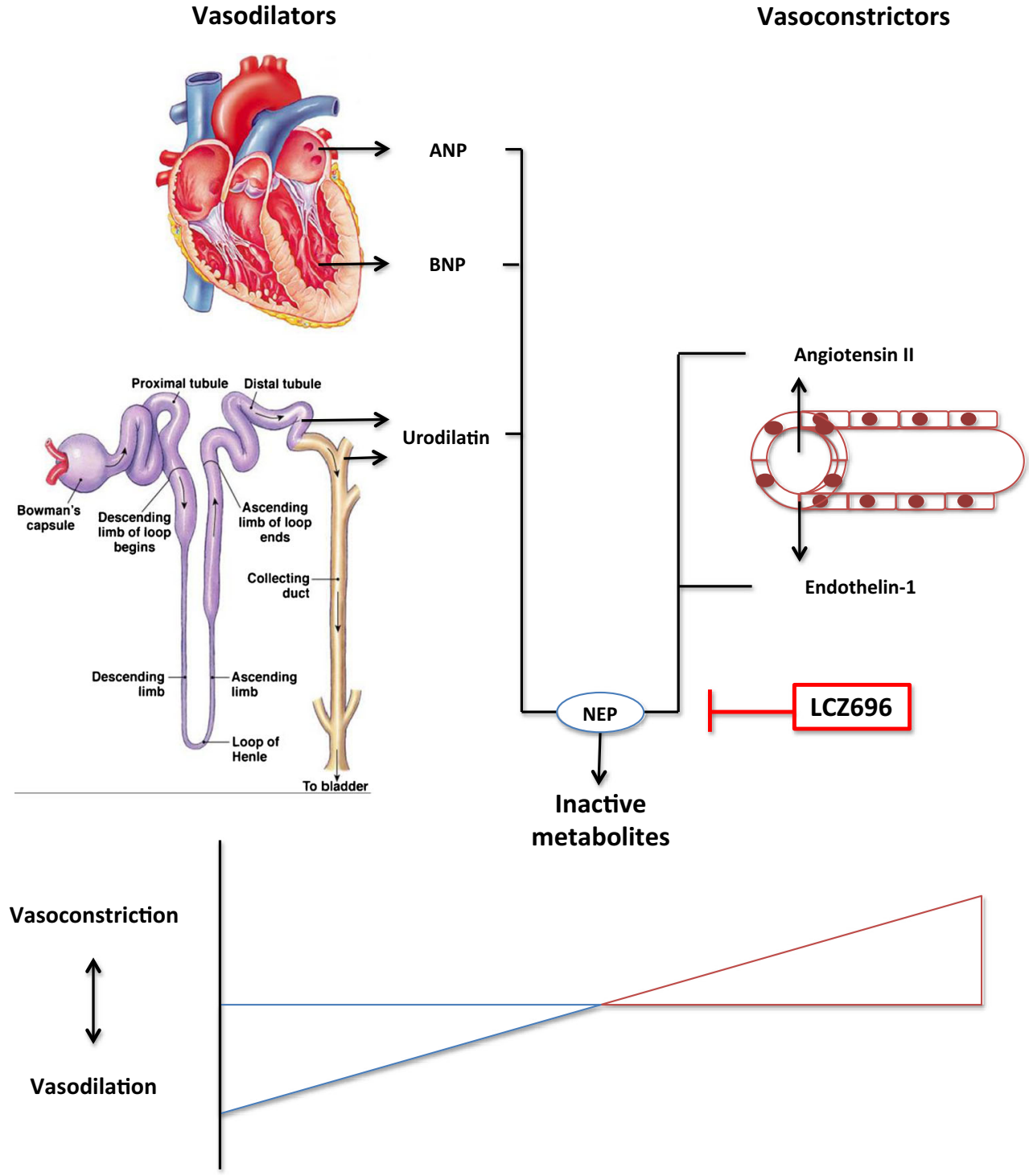

or if dual ACE/NEP inhibition had a stronger synergistic effect compared with ARNI.

In 2010, the first animal study using the ARNI LCZ696 was published. Gu et al. reported on the pharmacokinetics of LCZ696 in beagle dogs and its pharmacodynamics in rats [18]. Single oral administration of LCZ696 to rats transgenic for human renin and angiotensinogen caused a dosedependent decrease in mean arterial pressure (MAP) compared to baseline, while it increased plasma ANP concentration and immunoreactivity in Sprague-Dawley (SD) rats chronically infused with exogenous A-type (atrial) natriuretic peptide (ANP) [18]. LCZ696 was not compared with valsartan alone.

Von Lueder et al. compared LCZ696 with vehicle treatment in SD rats after experimental myocardial infarction [23]. As expected, after 4 weeks of treatment, LCZ696-treated rats had a lower blood pressure, a higher cardiac output, a lower heart weight and less cardiac fibrosis compared with vehicle treatment. In vitro, Von Lueder et al. assessed the effects of valsartan, AHU377, LBQ657 or valsartan + LBQ657 on rat neonatal cardiac myocytes and fibroblasts, after Ang II pretreatment. Valsartan and LBQ657 both inhibited Ang IIinduced cardiac myocyte hypertrophy when applied separately. When applied simultaneously, valsartan + LBQ657 only outperformed valsartan when a very low dose or a very high dose of valsartan was combined with LBQ657 (fixed dose). Valsartan decreased collagen accumulation in cardiac fibroblasts, while LBQ657 did not affect collagen accumulation. Dual treatment with valsartan + LBQ657 consistently inhibited Ang II-induced collagen accumulation more than valsartan alone [23]. Unfortunately, in this study, LCZ696 treatment was not compared with valsartan in vivo.

Bai et al. pretreated mice with LCZ696, valsartan or vehicle before inducing ischaemic brain damage by middle 
Table 1 Comparison of LCZ696 to other anti-hypertensive therapies or vehicle in animal models

\begin{tabular}{|c|c|c|c|c|c|}
\hline \multirow[t]{2}{*}{ Year } & \multirow[t]{2}{*}{ Author } & \multirow[t]{2}{*}{ Animal model } & \multirow[t]{2}{*}{ ARNI (ARB + NEPi) } & \multicolumn{2}{|c|}{ Comparison } \\
\hline & & & & $\mathrm{ARB}$ & Other \\
\hline 2008 & Pu et al. & SHRSP rats & Valsartan + CGS25354 & Valsartan & CGS30440 b \\
\hline 2010 & Gu et al. & Beagle dogs and SD rats & Valsartan + sacubitril ${ }^{\mathrm{a}}$ & - & - \\
\hline 2015 & $\begin{array}{l}\text { Von Lueder } \\
\text { et al. }\end{array}$ & $\mathrm{SD}$ rats & Valsartan + sacubitril $^{\mathrm{a}}$ & - & Vehicle \\
\hline 2015 & Bai et al. & C57BL/6J mice & Valsartan + sacubitril $^{\mathrm{a}}$ & Valsartan & Vehicle \\
\hline 2015 & $\begin{array}{l}\text { Roksnoer } \\
\text { et al. }\end{array}$ & TGR (mRen2) rats & Irbesartan + thiorphan & Irbesartan & $\begin{array}{c}\text { Thiorphan, } \\
\text { vehicle }\end{array}$ \\
\hline 2016 & $\begin{array}{l}\text { Suematsu } \\
\text { et al. }\end{array}$ & $\begin{array}{l}\text { C57BL/6J mice + STZ } \\
\text { diabetes }\end{array}$ & Valsartan + sacubitril $^{\text {a }}$ & Valsartan & Vehicle \\
\hline 2016 & $\begin{array}{l}\text { Roksnoer } \\
\text { et al. }\end{array}$ & $\begin{array}{l}\text { TGR (mRen2) rat }+ \text { STZ } \\
\text { diabetes }\end{array}$ & Irbesartan + thiorphan & Irbesartan & Vehicle \\
\hline
\end{tabular}

$A C E i$ angiotensin-converting enzyme inhibitor, $N E P i$ neprilysin inhibitor, SHRSP stroke-prone spontaneously hypertensive rats, $S D$ Sprague Dawley, $S T Z$ streptozotocin

${ }^{\text {a }}$ LCZ696

${ }^{\mathrm{b}}$ Dual ACEi/NEPi cerebral artery occlusion [24]. Despite the absence of a blood pressure difference between the three groups, LCZ696-treated mice had a smaller ischaemic area compared with valsartantreated mice. LCZ696 caused a significant rise in serum ANP levels, compared with valsartan. Both valsartan and LCZ696 induced an increase in serum renin activity and serum Ang II concentration [24]. However, when calculating the dose of LCZ696, the authors used equal weight ratios instead of equal molar ratios and did not take in account area under the curve data to provide similar exposure to valsartan $[18,25]$. The authors did not comment on the absence of a difference in blood pressure between the three treatment groups.

Suematsu et al. used tenfold higher dosages of valsartan and LCZ696, compared with Bai et al., in streptozotocintreated diabetic mice, after myocardial reperfusion injury [26]. At the end of the 4-week treatment period, only valsartan-treated animals had a significantly lower blood pressure than vehicle-treated animals. Both valsartan- and LCZ696treated animals had a lower heart weight/body ratio than vehicle-treated animals. LCZ696-treated animals displayed a better ejection fraction, less cardiac fibrosis and lower cardiac TGF- $\beta$ and ANP expression compared with vehicle-treated animals. How exactly LCZ696 exerted its beneficial effects on TGF- $\beta$, fibrosis and cardiac function, independently from blood pressure, remained unclear [26].

In a recent study by our group, ARB (irbesartan) and ARNI (irbesartan + NEP inhibitor thiorphan) were compared in diabetic, hypertensive rats. ARB and ARNI induced a similar blood pressure-lowering effect, but ARNItreated animals displayed a lower heart weight, less proteinuria and less focal segmental glomerulosclerosis as compared to ARB-treated animals [27]. When analysing the eyes of these diabetic animals, ARNI-treated animals also had less severe diabetic retinopathy [Qiuhong Li, University of Florida, Gainesville, USA, unpublished observations].

This led to the conclusion that ARNI exerts beneficial effects in the heart, kidney and eye independent of its blood pressure-lowering effect. Effects could not easily be explained on the basis of changes in RAAS activity, natriuretic peptides or cGMP. Future studies should address to what degree ARNI affects local concentrations of natriuretic peptides, e.g. in the kidney. Here, natriuretic peptides might improve renal haemodynamics, but also exert anti-inflammatory effects.

In an earlier study in nondiabetic hypertensive rats, we observed that the dose of the NEP inhibitor given on top of an ARB is highly critical [28 ]: when the NEP inhibitor was dosed too high, circulating endothelin-1 levels started to rise, and this resulted in an increase in renal sodium-hydrogen exchanger type 3 protein expression and an upregulation of constrictor vascular endothelin type B receptors. As a consequence, blood pressure rose and a beneficial effect on cardiac hypertrophy was no longer observed. Since NEP is capable of cleaving endothelin-1, these findings are not unexpected and emphasize the need to measure changes in endothelin- 1 in future ARNI studies.

\section{Human Studies}

\section{Effects in the General Hypertensive Population}

ARNI was compared to valsartan in a proof of concept trial, to study whether neprilysin inhibition on top of ARB indeed enhances blood pressure reduction (Table 2) [25]. The study included 1328 patients with mild to moderate uncomplicated 
Table 2 Main characteristics of LCZ696-treated patients and effects of LCZ696 treatment

\begin{tabular}{|c|c|c|c|c|}
\hline Study & Ruilope et al. & $\begin{array}{l}\text { McMurray et al. } \\
\text { (PARADIGM-HF) }\end{array}$ & $\begin{array}{l}\text { Solomon et al. } \\
\text { (PARAMOUNT) }\end{array}$ & Ito et al. \\
\hline LCZ696-treated patients $(n)$ & 168 & 4187 & 149 & 32 \\
\hline Main inclusion criterium & Hypertension & HFrEF & HFpEF & CKD \\
\hline Additional specifications & & LVEF $29.6 \%(6.1)$ & LVEF $58 \%(7.3)$ & Stage 3 or 4 \\
\hline Age (years) & $53(10.2)$ & $63.8(11.4)$ & $71(9.4)$ & $66(9.1)$ \\
\hline Sex (\% male) & 57 & 78 & 43 & 75 \\
\hline Race (\% black) & 8 & 5 & NP & 0 \\
\hline Dosage LCZ696 (mg) & 200 & 200 & 200 & $\begin{array}{l}200-400 \\
\quad \text { (titration) }\end{array}$ \\
\hline Dosing frequency (per day) & 1 & 2 & 2 & 1 \\
\hline Duration of treatment (weeks) & 8 & 116 & 36 & 8 \\
\hline \multicolumn{5}{|c|}{ Concomitant anti-hypertensive medication } \\
\hline$\beta$-blockers $(\%)$ & 0 & 93 & 79 & 0 \\
\hline Diuretics (\%) & 0 & 80 & 100 & 6 \\
\hline $\begin{array}{l}\text { Mineralocorticoid receptor } \\
\text { antagonists }(\%)\end{array}$ & 0 & 54 & 19 & 0 \\
\hline Calcium channel blockers (\%) & 0 & NP & NP & 38 \\
\hline \multicolumn{5}{|l|}{ Baseline blood pressure } \\
\hline Systolic (mmHg) & $156.8(12.0)$ & $122(15)$ & $\begin{array}{l}136 \\
\text { (IQR } 130 \text { to } 145)\end{array}$ & $151.6(10.3)$ \\
\hline Diastolic (mmHg) & $99.9(4.1)$ & NP & 79 & $86.9(10.8)$ \\
\hline \multicolumn{5}{|c|}{ Change compared to baseline blood pressure } \\
\hline Systolic (mmHg) & $-11.0(\mathrm{NP})^{* *}$ & NP & $-7.5(15.0)^{*}$ & $-20.5(11.3)^{\$}$ \\
\hline Diastolic (mmHg) & $-6.1(\mathrm{NP})^{*}$ & NP & $-5.1(10.8)^{*}$ & $-8.3(6.3)^{\$}$ \\
\hline Main outcome & $\begin{array}{l}\text { Blood pressure } \\
\text { reduction* }\end{array}$ & $\begin{array}{l}\text { CVD-mortality/HF-hospitalization } \\
\text { reduction }\end{array}$ & $\begin{array}{l}\text { Left atrial volume } \\
\text { reduction* }\end{array}$ & $\begin{array}{l}\text { Blood pressure } \\
\text { UACR } \\
\text { reduction }\end{array}$ \\
\hline
\end{tabular}

Data are represented as mean (SD), unless otherwise indicated. Statistical significance was calculated using a Student's $t$ test $(* P<0.01, * * P<0.001$ compared with valsartan-treated controls; ${ }^{\#} P<0.001$ compared with enalapril treated controls; ${ }^{\$} P<0.001$ compared to baseline)

$N P$ not provided

hypertension. At baseline, untreated mean sitting systolic blood pressure (SBP) averaged $155.7 \mathrm{mmHg}$ and mean sitting diastolic blood pressure (DBP) averaged $99.7 \mathrm{mmHg}$. Patients with diabetes, cardiac or renal disease were excluded. Treatment with 100, 200 or $400 \mathrm{mg}$ LCZ696 once daily was compared to respectively 80,160 or $320 \mathrm{mg}$ valsartan once daily (i.e. equipotent amounts of valsartan [18]), or placebo, for a treatment period of 8 weeks. Most of the treatment effect occurred in the first week after start of the study drug, and the effect was almost maximal at 4 weeks of treatment. Treatment with $200 \mathrm{mg}$ LCZ696 lowered mean sitting SBP with $11.0 \mathrm{mmHg}$ and mean sitting DBP with $6.1 \mathrm{mmHg}$. This is an additional reduction of $-5.28 \mathrm{mmHg}(95 \%$ $\mathrm{CI}-8.28$ to -2.28$)$ in SBP and $-2.97 \mathrm{mmHg}(95 \% \mathrm{CI}$ -4.88 to -1.07$)$ in DBP when compared to $160 \mathrm{mg}$ valsartan. LCZ696-treated patients responded 14\% more often to treatment, attested by either a blood pressure below $140 / 90 \mathrm{mmHg}$ or more than 20 or $10 \mathrm{mmHg}$ reduction in SBP or DBP, respectively. Additional ambulatory blood pressure measurements demonstrated that these reductions were maintained throughout the day, although responses were most pronounced during nighttime. These outcomes did not improve further with $400 \mathrm{mg}$ LCZ696 versus $320 \mathrm{mg}$ valsartan. Occurrence rate or severity of reported adverse events did not differ between LCZ696 and valsartan.

\section{Effects in Heart Failure Populations}

RAS blockade reduces cardiovascular and all-cause mortality as well as hospitalization for worsening heart failure in patients with reduced ejection fraction (HFrEF) [29]. ACEi has obtained a central position herein, since enalapril was shown to greatly improve outcome already 25 years ago [30, 31]. A 
recent meta-analysis of three trials that studied the combination of RAS and neprilysin inhibition in HFrEF patients demonstrated a $15 \%$ risk reduction for the composite endpoint of death or hospitalization when compared to ACEi alone [32]. Unfortunately, combination of ACEi and neprilysin inhibition (omapatrilat) is more likely to cause serious angioedema [33, 34], prompting discontinuation of drug development. Therefore, the effect of ARNI on HFrEF outcome was studied in the Prospective Comparison of ARNI With ACEi to Determine Impact on Global Mortality and Morbidity in Heart Failure (PARADIGM-HF) trial (Table 2) [35•]. This study included 8399 patients with a mean left ventricular ejection fraction (LVEF) of $29.5 \%$. Nearly all patients continued receiving other drugs known to improve survival. Patients were randomly assigned to receive $200 \mathrm{mg}$ LCZ696 twice daily or $10 \mathrm{mg}$ enalapril twice daily. The trial was stopped early with a mean follow-up duration of 27 months due to a benefit in favour of LCZ696. The risk for the composite primary endpoint of cardiovascular-related death or first hospitalization for worsening heart failure was reduced by $20 \%$ in LCZ696-treated patients. In addition, all-cause mortality was reduced by $16 \%$. Patients with lower LVEF had a $9 \%$ increased risk per five-point reduction of ejection fraction but benefitted equally from treatment when compared to patients with higher LVEF [36]. The beneficial effect on mortality is mainly due to a reduction in sudden death or death from progression of heart failure [37]. Progressive impairment of functional capacity due to nonfatal worsening of symptoms was assessed by the Kansas City Cardiomyopathy Questionnaire (KCCQ). This score improved in patients treated with LCZ696 and declined in patients treated with enalapril (mean difference between groups was 0.95 points on a scale of 1 to 100). However, patients were $34 \%$ less likely to visit the emergency room and fewer patients required intravenous drug therapy, rehospitalization or admission to an intensive care unit. Moreover, LCZ696-treated patients were $22 \%$ less likely to require surgical implantation of a left ventricular assist device (LVAD) or heart transplantation [38]. Concomitant hypertension was present in $71 \%$ of the study population. Mean SBP at baseline averaged $122 \pm 15 \mathrm{mmHg}$. LCZ696 treatment lowered SBP with an additional $3.2 \pm 0.4 \mathrm{mmHg}$ when compared to enalapril. Consequently, LCZ696-treated patients were more likely to develop symptomatic hypotension (14 versus $9.2 \%$ ). In addition, more cases of angioedema were reported (19 versus 10 patients), although this difference was not significant. Conversely, patients treated with enalapril were more likely to develop hyperkalaemia or cough. Currently, the European Society of Cardiology (ESC) recommends the use of sacubitril/valsartan instead of ACEi in ambulatory patients who remain symptomatic despite optimal therapy [39].

Almost 50\% of heart failure patients have a preserved ejection fraction (HFpEF). RAS inhibition is less beneficial in this group of patients. Although it reduces the risk of hospitalization due to worsening of symptoms with $12 \%$, there are no improvements in cardiovascular or all-cause mortality [29]. Efficacy and safety of LCZ696 treatment in HFpEF patients was studied in Prospective Comparison of ARNI with ARB on Management of Heart Failure with Preserved Ejection Fraction (PARAMOUNT) (Table 2) [40]. In this study, 301 patients with a mean LVEF of 58\% were included. At baseline, most patients were treated for hypertension $(93.5 \%)$ and mean blood pressure averaged 136/79 mmHg. Patients were randomly assigned to $200 \mathrm{mg}$ LCZ696 twice daily or $160 \mathrm{mg}$ valsartan twice daily for a treatment period of 36 weeks. A significantly greater degree of blood pressure reduction was observed in LCZ696-treated patients than in valsartan-treated patients $(-7.5( \pm 15)$ versus $-1.5( \pm 16) \mathrm{mmHg}$ for mean sitting SBP; $-5.1( \pm 10.8)$ versus $-0.34( \pm 11.5) \mathrm{mmHg}$ for mean sitting DBP). Ventricular wall stretch induces production of proB-type natriuretic peptide (proBNP). N-terminal pro-B-type natriuretic peptide (NT-proBNP) is the inactive side product yielded upon cleavage of proBNP into biologically active BNP. Elevated serum NT-proBNP is a well-known marker for heart failure severity, as it is associated with increased risk of mortality and hospitalization [41]. NT-proBNP is not degraded by NEP and as such has a longer half-life than BNP. A decrease in NT-proBNP levels suggests reduced proBNP production due to reduced cardiac wall tension (Fig. 2), while an increase in BNP either suggests the opposite or might be due to NEP inhibition. In HFrEF patients, LCZ696 reduced NT-

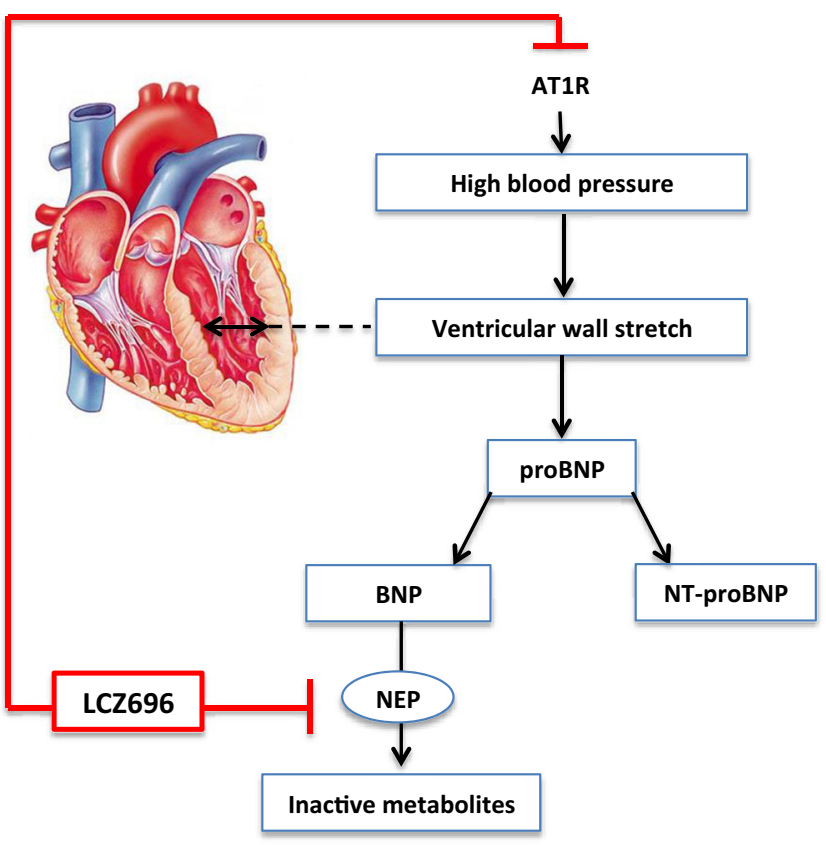

Fig. 2 Effect of LCZ696 on BNP and NT-proBNP production. LCZ696 lowers blood pressure by simultaneous blockade of angiotensin II type 1 receptors (AT1R) and inhibition of neprilysin (NEP). Relative dominance of either effect determines BNP levels. NT-proBNP represents effects on ventricular wall stretch, as it is not degraded by neprilysin 
proBNP and increased BNP [38]. In HFpEF patients, LCZ696 also reduced NT-proBNP at 12 weeks of treatment [40]. At 36 weeks, this difference was no longer significant. However, at this time point, left atrial width (mean difference $0.7 \mathrm{~mm}$ ) and volume (mean difference $4.97 \mathrm{~mL}$ ) were reduced in favour of LCZ696. These effects were independent of blood pressure lowering [42]. Effect of ARNI treatment on mortality and hospitalization in HFpEF patients is currently being studied in Prospective Comparison of ARNI with ARB Global Outcomes in Heart Failure with Preserved Ejection Fraction (PARAGON-HF).

\section{Effects in Other High-Risk Populations}

CKD patients are at high risk for cardiovascular morbidity and mortality, added on top of the risk of progression towards ESRD. Diabetes and hypertension are the most common causes of CKD worldwide and additionally independent risk factors for cardiovascular complications. Simultaneous presence of all three risk factors is not uncommon. However, the complex interplay between these diseases does appear to alter treatment responses. For HFrEF patients with renal function decline, regardless of cause and comorbidities, benefit of treatment with LCZ696 was equal for patients with eGFR $\geq 60 \mathrm{~mL} /$ $\mathrm{min} / 1.73 \mathrm{~m}^{2}$ and reduced eGFR (between 30 and $60 \mathrm{~mL} / \mathrm{min} /$ $1.73 \mathrm{~m}^{2}$ ) [36]. However, treatment of diabetic HFrEF patients with $200 \mathrm{mg}$ LCZ696 twice daily did not reduce cardiovascular and all-cause mortality when compared to valsartan, although risk for heart failure hospitalization was improved, comparable to that of nondiabetic patients [43]. Interestingly, LCZ696 reduced NT-proBNP levels particularly in HFpEF patients with diabetes, indicating a more pronounced reduction of ventricular wall tension when compared to nondiabetic patients [40]. Furthermore, LCZ696 may prevent progression of renal damage especially in patients with diabetic nephropathy. LCZ696 reduced urinary albumin-to-creatinine ratio (UACR) by $15.1 \%$ in patients with hypertension and moderate to severe renal impairment, with a greater effect in patients with macroalbuminuria than in micro- or normoalbuminuria [44•]. Conversely, a small but significant increase in UACR (2.4 to $2.9 \mathrm{mg} / \mathrm{mmol}$ ) was observed in LCZ696-treated HFpEF patients [45], despite the fact that eGFR was better preserved after 36 weeks of treatment $(-1.6$ versus $-5.2 \mathrm{~mL} /$ $\min / 1.73 \mathrm{~m}^{2}$ ) [41]. Increased albuminuria in these patients might be caused by ANP-induced relaxation or antiproliferative effects in renal mesangial cells [46, 47], in combination with impaired proximal tubular protein reabsorption [48]. No significant differences in renal outcome were found between patients with HFrEF treated with either LCZ696 or enalapril, although numerically fewer patients developed ESRD with LCZ696 ( 8 versus 16 patients, $P=0.11$ ). These favourable outcomes might be explained by a reduction of renal tubulointerstitial fibrosis and focal segmental glomerulosclerosis, as observed in ARNI-treated diabetic, hypertensive rats when compared to ARB-treated controls [27]. Moreover, LBQ657 reduced the Ang II-stimulated increase in collagen synthesis in renal mesangial cells [49]. These effects occurred in a blood pressure-independent manner.

Despite these potential advantages in renal outcome, there may be some limitations to the use of LCZ696 in CKD patients. Sacubitrilat is primarily eliminated by the kidneys. The degree of decline in eGFR correlated with increased maximum plasma drug concentration and half-life, as the percentage of sacubitrilat excreted in urine decreased progressively. Therefore, normotensive patients with moderate to severe renal impairment more often developed orthostatic hypotension [20]. In PARADIGM-HF, blood pressure was well-controlled due to the continued use of several classes of antihypertensive drugs, and mean SBP at baseline averaged $122 \pm 15 \mathrm{mmHg}$ (Table 2) [35•]. Consequently, hypotension occurred more often in patients that were subsequently exposed to LCZ696 and was the most common reason for dose reduction, whereas a higher serum creatinine level was the main predictor of this phenomenon. This indicates that indeed normotensive patients with reduced renal function are at higher risk of developing hypotension. However, hypertension is both a cause and consequence of CKD and blood pressure is often poorly controlled in these patients [50]. Therefore, the safety and efficacy of LCZ696 was assessed in 32 Japanese patients with hypertension and CKD stage $3(78.1 \%)$ or stage $4(21.9 \%)$ (Table 2) [44•]. Initial treatment with $100 \mathrm{mg}$ LCZ696 once daily reduced mean sitting blood pressure to $130 / 80 \mathrm{mmHg}$ or lower in $19 \%$ of the population. The dosage in other patients was increased to either $200 \mathrm{mg}$ (25\%) or $400 \mathrm{mg}$ (56\%) LCZ696 once daily, for a total treatment duration of 8 weeks. The blood pressure-lowering effect occurred mostly in the first week of treatment and was maximal at 4 weeks. Stage 3 CKD patients had a mean blood pressure of 152.1/87.7 $\mathrm{mmHg}$ at baseline. With LCZ696, 60\% of the patients had an adequate SBP reduction of $\geq 20 \mathrm{mmHg}$ or values $\leq 130 \mathrm{mmHg}$ and in $76 \%$ of the patients an adequate DBP reduction of $\geq 10 \mathrm{mmHg}$ or values $\leq 80 \mathrm{mmHg}$. Mean reduction in all patients was 21.3/9.1 mmHg. LCZ696 treatment was also effective in stage $4 \mathrm{CKD}$ patients. Their mean baseline blood pressure of $149.8 / 83.9 \mathrm{mmHg}$ was reduced by $17.7 /$ $5.5 \mathrm{mmHg}$. Adequate SBP and DBP responses were obtained in $57 \%$ of the patients. No events of hypotension were reported in either CKD class, although plasma doses of LBQ657 tended to increase with decreasing renal function. The safety of LCZ696 treatment in CKD patients and effects on renal function 
are currently being studied in the United Kingdom Heart and Renal Protection III (HARP-III) trial [51].

\section{Limitations}

NEP is involved in the degradation of many peptides, among which amyloid beta 1-42 $\left(\mathrm{A} \beta_{1-42}\right)$. Intracerebral infusion of the NEP inhibitor thiorphan induces hippocampal accumulation and deposition of $A \beta_{1-42}$ in rats, already at in vivo concentrations of $2.5 \mathrm{ng} / \mathrm{mL}$ [52]. Aggregation of $A \beta_{1-42}$ is believed to play a critical role in the development of Alzheimer's disease (reviewed in [53]). Blood-brain barrier penetration of LBQ657 was assessed in 17 healthy volunteers treated with $400 \mathrm{mg}$ LCZ696 once daily [54]. After 2 weeks of treatment, a mean maximum drug concentration of $19.2( \pm 11.3) \mathrm{ng} /$ $\mathrm{mL}$ was found in cerebrospinal fluid (CSF). There was no effect on CSF $A \beta_{1-42}$ concentration when LCZ696treated individuals were compared to placebo-treated controls. Differences in plasma $A \beta_{1-42}$ concentration were not determined, although plasma $A \beta_{1-40}$ concentration increased by $50 \%$. These results do not dispel all concerns. CSF clearance of $A \beta$ appears to be a minor route, as the blood-brain barrier traffics most of the brain $A \beta$ directly out into the peripheral circulation [55]. Additionally, the measurements in placebo-treated controls demonstrate high variability of CSF $A \beta_{1-42}$ concentration. Therefore, these results do not rule out the possibility of accumulation $\mathrm{A} \beta_{1-42}$ in the brain, which would go undetected by CSF measurements. Additionally, hypertension and diabetes are known to contribute to blood-brain barrier dysfunction, rendering these patients more vulnerable, as NEP inhibition might induce $A \beta_{1-42}$ in a dose-dependent manner [56]. However, these risks might be more relevant for diseases with greater longevity than HFrEF.

\section{Conclusions}

In summary, when compared to ARB, ARNI superiorly reduces blood pressure in uncomplicated, essential hypertension as well as in heart failure and CKD populations. When compared to ACEi, ARNI superiorly prevents death and hospitalization in HFrEF patients that already receive all other medication known to improve outcome. ARNI appears to improve renal outcome, particularly in high-risk, multi-morbid populations. Although patients with concomitant heart failure and diabetes show no reduction in mortality, they do demonstrate reverse left atrial remodeling, less heart failure hospitalization and reduced albuminuria. Preclinical data in hypertensive, diabetic rats additionally suggests blood pressureindependent improvement of renal damage, attested by decreased proteinuria and abated glomerulosclerosis. ARNI might mediate these effects by impeding activation of the proinflammatory cytokine TGF- $\beta$, which plays a central role in the fibrotic response and accumulation of collagen. This could explain the improved renal outcomes but requires further experimental study. Importantly, ARB and ARNI have not yet been compared in patients with CKD due to diabetes and hypertension, and thus the results of the HARP-III trial are anxiously awaited [51]. Until that moment, the application of ARNI as a renoprotective drug for this high-risk patient population is hampered. Moreover, sacubitrilat, the active neprilysin-inhibiting component, is eliminated by the kidney. Sacubitrilat-exposed normotensive patients with impaired renal function can therefore, due to increased exposure, develop hypotension. This is less likely to be a problem for the hypertensive population. Caution is advised regarding the risk of cerebral $A \beta_{1-}$ 42 accumulation and Alzheimer's disease in populations with a longer median survival. Finally, we need additional insight into the mechanism of action of ARNI. Although a decrease in NTproBNP and a rise in BNP have been reported [38], this might be an assay artefact. Indeed, ARNI may promote peptide glycosylation, thereby affecting not only proBNP cleavage but also the assays of NT-proBNP and BNP: NT-proBNP would become invisible, while proBNP would additionally be detected in BNP assays [57 ]. Another complicating factor is that BNP of all natriuretic peptides is the least susceptible to degradation by NEP and as such acts as an endogenous inhibitor of NEP [58]. If so, patients with elevated BNP levels already undergo NEP inhibition and might respond less or not at all to LCZ696.

\section{Compliance with Ethical Standards}

Conflict of Interest Drs. Uijl, Roksnoer, Hoorn, and Danser declare no conflicts of interest relevant to this manuscript.

Human and Animal Rights and Informed Consent This article does not contain any studies with human or animal subjects performed by any of the authors.

Open Access This article is distributed under the terms of the Creative Commons Attribution 4.0 International License (http:// creativecommons.org/licenses/by/4.0/), which permits unrestricted use, distribution, and reproduction in any medium, provided you give appropriate credit to the original author(s) and the source, provide a link to the Creative Commons license, and indicate if changes were made.

\section{References}

Papers of particular interest, published recently, have been highlighted as:

- Of importance

1. Mortality GBD. Causes of death C. Global, regional, and national age-sex specific all-cause and cause-specific mortality for 240 causes of death, 1990-2013: a systematic analysis for the global burden of disease study 2013. Lancet. 2015;385:117-71.

2. Lim SS, Vos T, Flaxman AD, Danaei G, Shibuya K, Adair-Rohani $\mathrm{H}$, et al. A comparative risk assessment of burden of disease and 
injury attributable to 67 risk factors and risk factor clusters in 21 regions, 1990-2010: a systematic analysis for the global burden of disease study 2010. Lancet. 2012;380:2224-60.

3. Foley RN, Parfrey PS, Sarnak MJ. Clinical epidemiology of cardiovascular disease in chronic renal disease. Am J Kidney Dis. 1998;32:S112-9.

4. Colosia AD, Palencia R, Khan S. Prevalence of hypertension and obesity in patients with type 2 diabetes mellitus in observational studies: a systematic literature review. Diabetes Metab Syndr Obes. 2013;6:327-38.

5. Adler AI, Stratton IM, Neil HA, Yudkin JS, Matthews DR, Cull CA, et al. Association of systolic blood pressure with macrovascular and microvascular complications of type 2 diabetes (UKPDS 36): prospective observational study. BMJ. 2000;321: 412-9.

6. Ettehad D, Emdin CA, Kiran A, Anderson SG, Callender T, Emberson J, et al. Blood pressure lowering for prevention of cardiovascular disease and death: a systematic review and meta-analysis. Lancet. 2016;387:957-67.

7. Palmer SC, Mavridis D, Navarese E, Craig JC, Tonelli M, Salanti $\mathrm{G}$, et al. Comparative efficacy and safety of blood pressurelowering agents in adults with diabetes and kidney disease: a network meta-analysis. Lancet. 2015;385:2047-56.

8. James PA, Oparil S, Carter BL, Cushman WC, DennisonHimmelfarb C, Handler J, et al. Evidence-based guideline for the management of high blood pressure in adults: report from the panel members appointed to the eighth joint national committee (JNC 8). JAMA. 2014;311:507-20.

9. Makani H, Bangalore S, Desouza KA, Shah A, Messerli FH. Efficacy and safety of dual blockade of the renin-angiotensin system: meta-analysis of randomised trials. BMJ. 2013;346:f360.

10. Balcarek J, Seva Pessoa B, Bryson C, Azizi M, Menard J, Garrelds IM, et al. Multiple ascending dose study with the new renin inhibitor VTP-27999: nephrocentric consequences of too much renin inhibition. Hypertension. 2014;63:942-50.

11. Packer M. Why do the kidneys release renin in patients with congestive heart failure? A nephrocentric view of converting-enzyme inhibition. Am J Cardiol. 1987;60:179-84.

12. Lange S, Fraune C, Alenina N, Bader M, Danser AH, Frenay AR, et al. Aliskiren accumulation in the kidney: no major role for binding to renin or prorenin. J Hypertens. 2013;31:713-9.

13. Gee NS, Kenny AJ. Proteins of the kidney microvillar membrane. The $130 \mathrm{kDa}$ protein in pig kidney, recognized by monoclonal antibody $\mathrm{GK} 5 \mathrm{C} 1$, is an ectoenzyme with aminopeptidase activity. Biochem J. 1985;230:753-64.

14. Schulz WW, Hagler HK, Buja LM, Erdos EG. Ultrastructural localization of angiotensin I-converting enzyme (EC 3.4.15.1) and neutral metalloendopeptidase (EC 3.4.24.11) in the proximal tubule of the human kidney. Lab Invest. 1988;59:789-97.

15. Ando S, Rahman MA, Butler GC, Senn BL, Floras JS. Comparison of candoxatril and atrial natriuretic factor in healthy men. Effects on hemodynamics, sympathetic activity, heart rate variability, and endothelin. Hypertension. 1995;26:1160-6.

16. Unger $\mathrm{T}, \mathrm{Li}$ J. The role of the renin-angiotensin-aldosterone system in heart failure. J Renin-Angiotensin-Aldosterone Syst. 2004;5 Suppl 1:S7-10.

17. Han B, Hasin Y. Cardiovascular effects of natriuretic peptides and their interrelation with endothelin-1. Cardiovasc Drugs Ther. 2003;17:41-52.

18. Gu J, Noe A, Chandra P, Al-Fayoumi S, Ligueros-Saylan M, Sarangapani R, et al. Pharmacokinetics and pharmacodynamics of LCZ696, a novel dual-acting angiotensin receptor-neprilysin inhibitor (ARNi). J Clin Pharmacol. 2010;50:401-14.

19. Flarakos J, Du Y, Bedman T, Al-Share Q, Jordaan P, Chandra P, et al. Disposition and metabolism of [(14)C] sacubitril/valsartan (formerly LCZ696) an angiotensin receptor neprilysin inhibitor, in healthy subjects. Xenobiotica. 2016;46:986-1000.

20. Ayalasomayajula SP, Langenickel TH, Jordaan P, Zhou W, Chandra $\mathrm{P}$, Albrecht D, et al. Effect of renal function on the pharmacokinetics of LCZ696 (sacubitril/valsartan), an angiotensin receptor neprilysin inhibitor. Eur J Clin Pharmacol. 2016;72:1065-73.

21. Waldmeier F, Flesch G, Muller P, Winkler T, Kriemler HP, Buhlmayer P, et al. Pharmacokinetics, disposition and biotransformation of [14C]-radiolabelled valsartan in healthy male volunteers after a single oral dose. Xenobiotica. 1997;27:59-71.

22. $\mathrm{Pu} \mathrm{Q}$, Brassard $\mathrm{P}$, Javeshghani DM, Iglarz M, Webb RL, Amiri F, et al. Effects of combined AT1 receptor antagonist/NEP inhibitor on vascular remodeling and cardiac fibrosis in SHRSP. J Hypertens. 2008;26:322-33.

23. von Lueder TG, Wang BH, Kompa AR, Huang L, Webb R, Jordaan P, et al. Angiotensin receptor neprilysin inhibitor LCZ696 attenuates cardiac remodeling and dysfunction after myocardial infarction by reducing cardiac fibrosis and hypertrophy. Circ Heart Fail. 2015;8:71-8.

24. Bai HY, Mogi M, Nakaoka H, Kan-No H, Tsukuda K, Chisaka T, et al. Pre-treatment with LCZ696, an orally active angiotensin receptor neprilysin inhibitor, prevents ischemic brain damage. Eur J Pharmacol. 2015;762:293-8.

25. Ruilope LM, Dukat A, Bohm M, Lacourciere Y, Gong J, Lefkowitz MP. Blood-pressure reduction with LCZ696, a novel dual-acting inhibitor of the angiotensin II receptor and neprilysin: a randomised, double-blind, placebo-controlled, active comparator study. Lancet. 2010;375:1255-66.

26. Suematsu Y, Miura S, Goto M, Matsuo Y, Arimura T, Kuwano T, et al. LCZ696, an angiotensin receptor-neprilysin inhibitor, improves cardiac function with the attenuation of fibrosis in heart failure with reduced ejection fraction in streptozotocin-induced diabetic mice. Eur J Heart Fail. 2016;18:386-93.

27. Roksnoer LC, van Veghel R, van Groningen MC, de Vries R, Garrelds IM, Bhaggoe UM, et al. Blood pressure-independent renoprotection in diabetic rats treated with AT1 receptorneprilysin inhibition compared with AT1 receptor blockade alone. Clin Sci (Lond). 2016;130:1209-20.

28. Roksnoer LC, Van Veghel R, De Vries R, Garrelds IM, Bhaggoe UM, Friesema EC, et al. Optimum AT1 receptor-neprilysin inhibition has superior cardioprotective effects compared with AT1 receptor blockade alone in hypertensive rats. Kidney Int. 2015;88: 109-20. This study demonstrates that the degree of NEPi is critical: if dosed too high, NEPi can induce a rise in circulating endothelin-1 levels and consequently, a rise in blood pressure.

29. Emdin CA, Callender T, Cao J, McMurray JJ, Rahimi K. Metaanalysis of large-scale randomized trials to determine the effectiveness of inhibition of the renin-angiotensin aldosterone system in heart failure. Am J Cardiol. 2015;116:155-61.

30. Effects of enalapril on mortality in severe congestive heart failure. Results of the Cooperative North Scandinavian Enalapril Survival Study (CONSENSUS). The CONSENSUS trial study group. N Engl J Med. 1987;316:1429-35.

31. Effect of enalapril on survival in patients with reduced left ventricular ejection fractions and congestive heart failure. The SOLVD investigators. N Engl J Med. 1991;325:293-302.

32. Solomon SD, Claggett B, McMurray JJ, Hernandez AF, Fonarow GC. Combined neprilysin and renin-angiotensin system inhibition in heart failure with reduced ejection fraction: a meta-analysis. Eur J Heart Fail. 2016;18:1238-43.

33. Packer M, Califf RM, Konstam MA, Krum H, McMurray JJ, Rouleau JL, et al. Comparison of omapatrilat and enalapril in patients with chronic heart failure: the omapatrilat versus enalapril randomized trial of utility in reducing events (OVERTURE). Circulation. 2002;106:920-6. 
34. Kostis JB, Packer M, Black HR, Schmieder R, Henry D, Levy E. Omapatrilat and enalapril in patients with hypertension: the omapatrilat cardiovascular treatment vs. Enalapril (OCTAVE) trial. Am J Hypertens. 2004;17:103-11.

35. McMurray JJ, Packer M, Desai AS, Gong J, Lefkowitz MP, Rizkala AR, et al. Angiotensin-neprilysin inhibition versus enalapril in heart failure. N Engl J Med. 2014;371:993-1004. This study demonstrates that ARNI greatly reduces the risk of death and hospitalization in heart failure patients with reduced ejection fraction when compared to current golden standard treatment.

36. Solomon SD, Claggett B, Desai AS, Packer M, Zile M, Swedberg $\mathrm{K}$, et al. Influence of ejection fraction on outcomes and efficacy of sacubitril/valsartan (LCZ696) in heart failure with reduced ejection fraction: the prospective comparison of ARNI with ACEI to determine impact on global mortality and morbidity in heart failure (PARADIGM-HF) trial. Circ Heart Fail. 2016;9, e002744.

37. Desai AS, McMurray JJ, Packer M, Swedberg K, Rouleau JL, Chen $\mathrm{F}$, et al. Effect of the angiotensin-receptor-neprilysin inhibitor LCZ696 compared with enalapril on mode of death in heart failure patients. Eur Heart J. 2015;36:1990-7.

38. Packer M, McMurray JJ, Desai AS, Gong J, Lefkowitz MP, Rizkala AR, et al. Angiotensin receptor neprilysin inhibition compared with enalapril on the risk of clinical progression in surviving patients with heart failure. Circulation. 2015;131:54-61.

39. Ponikowski P, Voors AA, Anker SD, Bueno H, Cleland JG, Coats AJ, et al. ESC Guidelines for the diagnosis and treatment of acute and chronic heart failure: The Task Force for the diagnosis and treatment of acute and chronic heart failure of the European Society of Cardiology (ESC). Developed with the special contribution of the Heart Failure Association (HFA) of the ESC. Eur J Heart Fail. 2016;18:891-975.

40. Solomon SD, Zile M, Pieske B, Voors A, Shah A, Kraigher-Krainer E, et al. The angiotensin receptor neprilysin inhibitor LCZ696 in heart failure with preserved ejection fraction: a phase 2 doubleblind randomised controlled trial. Lancet. 2012;380:1387-95.

41. Anand IS, Rector TS, Cleland JG, Kuskowski M, McKelvie RS, Persson $\mathrm{H}$, et al. Prognostic value of baseline plasma aminoterminal pro-brain natriuretic peptide and its interactions with irbesartan treatment effects in patients with heart failure and preserved ejection fraction: findings from the I-PRESERVE trial. Circ Heart Fail. 2011;4:569-77.

42. Jhund PS, Claggett B, Packer M, Zile MR, Voors AA, Pieske B, et al. Independence of the blood pressure lowering effect and efficacy of the angiotensin receptor neprilysin inhibitor, LCZ696, in patients with heart failure with preserved ejection fraction: an analysis of the PARAMOUNT trial. Eur J Heart Fail. 2014;16:671-7.

43. Kristensen SL, Preiss D, Jhund PS, Squire I, Cardoso JS, Merkely B et al. Risk related to pre-diabetes mellitus and diabetes mellitus in heart failure with reduced ejection fraction: insights from Prospective Comparison of ARNI with ACEI to Determine Impact on Global Mortality and Morbidity in Heart Failure Trial. Circ Heart Fail. 2016;9.

44. Ito S, Satoh M, Tamaki Y, Gotou H, Charney A, Okino N, et al. Safety and efficacy of LCZ696, a first-in-class angiotensin receptor neprilysin inhibitor, in Japanese patients with hypertension and renal dysfunction. Hypertens Res. 2015;38:269-75. This study is the first to demonstrate that ARNI exerts beneficial effects beyond blood pressure lowering: ARNI can reduce albuminuria and thus improve renal outcome in hypertensive, CKD patients.

45. Voors AA, Gori M, Liu LC, Claggett B, Zile MR, Pieske B, et al. Renal effects of the angiotensin receptor neprilysin inhibitor LCZ696 in patients with heart failure and preserved ejection fraction. Eur J Heart Fail. 2015;17:510-7.

46. Marin-Grez M, Fleming JT, Steinhausen M. Atrial natriuretic peptide causes pre-glomerular vasodilatation and post-glomerular vasoconstriction in rat kidney. Nature. 1986;324:473-6.

47. Pedersen MM, Christiansen JS, Pedersen EB, Mogensen CE. Determinants of intra-individual variation in kidney function in normoalbuminuric insulin-dependent diabetic patients: importance of atrial natriuretic peptide and glycaemic control. Clin Sci (Lond). 1992;83:445-51.

48. Jacobs EM, Vervoort G, Branten AJ, Klasen I, Smits P, Wetzels JF. Atrial natriuretic peptide increases albuminuria in type I diabetic patients: evidence for blockade of tubular protein reabsorption. Eur J Clin Investig. 1999;29:109-15.

49. Wang BH, von Lueder TG, Kompa AR, Huang L, Webb R, Jordaan $\mathrm{P}$, et al. Combined angiotensin receptor blockade and neprilysin inhibition attenuates angiotensin-II mediated renal cellular collagen synthesis. Int J Cardiol. 2015;186:104-5.

50. Sarafidis PA, Sharpe CC, Wood E, Blacklock R, Rumjon A, AlYassin A, et al. Prevalence, patterns of treatment, and control of hypertension in predialysis patients with chronic kidney disease. Nephron Clin Pract. 2012;120:c147-55.

51. Group UH-IC. Randomized multicentre pilot study of sacubitril/ valsartan versus irbesartan in patients with chronic kidney disease: United Kingdom Heart and Renal Protection (HARP)- III-rationale, trial design and baseline data. Nephrol Dial Transplant. 2016.

52. Iwata N, Tsubuki S, Takaki Y, Watanabe K, Sekiguchi M, Hosoki E, et al. Identification of the major Abeta1-42-degrading catabolic pathway in brain parenchyma: suppression leads to biochemical and pathological deposition. Nat Med. 2000;6:143-50.

53. Karran E, Mercken M, De Strooper B. The amyloid cascade hypothesis for Alzheimer's disease: an appraisal for the development of therapeutics. Nat Rev Drug Discov. 2011;10:698-712.

54. Langenickel TH, Tsubouchi C, Ayalasomayajula S, Pal P, Valentin MA, Hinder M, et al. The effect of LCZ696 (sacubitril/valsartan) on amyloid-beta concentrations in cerebrospinal fluid in healthy subjects. Br J Clin Pharmacol. 2016;81:878-90.

55. Qosa H, Abuasal BS, Romero IA, Weksler B, Couraud PO, Keller $\mathrm{JN}$, et al. Differences in amyloid-beta clearance across mouse and human blood-brain barrier models: kinetic analysis and mechanistic modeling. Neuropharmacology. 2014;79:668-78.

56. Iwata N, Tsubuki S, Takaki Y, Shirotani K, Lu B, Gerard NP, et al. Metabolic regulation of brain Abeta by neprilysin. Science. 2001;292:1550-2.

57. Rosjo H, Dahl MB, Jorgensen M, Roysland R, Brynildsen J, Cataliotti A, et al. Influence of glycosylation on diagnostic and prognostic accuracy of $\mathrm{N}$-terminal pro-B-type natriuretic peptide in acute dyspnea: data from the Akershus cardiac examination 2 study. Clin Chem. 2015;61:1087-97. This study critically addresses the measurements of BNP and NT-proBNP.

58. Vodovar N, Seronde MF, Laribi S, Gayat E, Lassus J, Januzzi Jr JL, et al. Elevated plasma B-type natriuretic peptide concentrations directly inhibit circulating neprilysin activity in heart failure. JACC Heart Fail. 2015;3:629-36. 